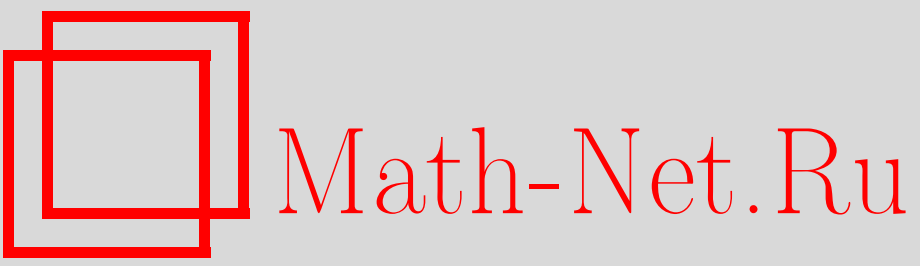

Ю. М. Волчков, Вариационное уравнение в двухслойной модели оболочки Работнова и критическое время выпучивания подкрепленных оболочек при ползучести, Вестн. Сам. гос. техн. ун-та. Сер. Физ.-мат. науки, 2010, выпуск 5()$, 72-78$

DOI: https://doi.org/10.14498/vsgtu790

Использование Общероссийского математического портала Math-Net.Ru подразумевает, что вы прочитали и согласны с пользовательским соглашением http: //www. mathnet.ru/rus/agreement

Параметры загрузки:

IP : 107.22 .136 .117

26 апреля 2023 г., 12:18:23 
УДК 539.376

\title{
ВАРИАЦИОННОЕ УРАВНЕНИЕ В ДВУХСЛОЙНОЙ МОДЕЛИ ОБОЛОЧКИ РАБОТНОВА И КРИТИЧЕСКОЕ ВРЕМЯ ВЫПУЧИВАНИЯ ПОДКРЕПЛЕННЫХ ОБОЛОЧЕК ПРИ ПОЛЗУЧЕСТИ
}

\section{Ю. М. Волчков}

Институт гидродинамики им. М. А. Лаврентьева СО РАН, 630090, Новосибирск, пр-т Академика Лаврентьева, 15.

E-mail: volk@hydro.nsc.ru

\begin{abstract}
Излагается процедура вычисления критического времени выпучивания оболочек при ползучести с использованием вариационного уравнения Ю. Н. Работнова. Приводится сравнение критических времен для подкрепленных оболочек, вычисленных с использованием трехслойной модели оболочки, с экспериментальными данными и результатами других авторов.
\end{abstract}

Ключевые слова: двухслойная и трехслойная модели оболочки, ползучесть, критическое время.

Введение. В работе [1] Ю.Н. Работновым для решения задач упругопластического деформирования предложена двухслойная модель оболочки. В работах $[2,3]$ модель была обобщена на случай деформирования оболочек в условиях ползучести. В [3] указан также класс оболочек, для которых применение двухслойной модели не может вносить больших погрешностей при решении конкретных задач. Этот класс оболочек описывается так называемой технической теорией оболочек, которая включает теорию осесимметричной деформации круговой цилиндрической оболочки, теорию длинных цилиндрических оболочек и теорию чистого изгиба криволинейной трубы. Применение двухслойной модели оболочки позволило разработать эффективный численный метод решения задач о напряженно-деформированном состоянии оболочек в условиях ползучести [4]. Обобщение двухслойной модели на случай подкрепленных ребрами оболочек дано в работах $[5,6]$.

1. Двухслойная модель оболочки Работнова. При построении упрощенной теории оболочек реальная оболочка толщины $2 H$ заменяется двухслойной моделью - оболочкой, состоящей из двух слоев толщиной $h_{1}$ каждый, расстояние между серединами которых равно $2 h$ (рис. 1 ). Слои считаются связанными между собой таким образом, что связь передает сдвигающее усилие,но не воспринимает растягивающих усилий и изгибающих моментов. Таким образом, деформация слоев происходит в соответствии с гипотезой КирхгофаЛява. За отсчетную поверхность принимается поверхность, находящаяся посередине между слоями. В силу малости толщины несущих слоев изменением деформаций в пределах их толщин можно пренебречь. Выполнение условия $h_{1} \ll h$ можно обеспечить соответствующим выбором констант в законах ползучести для реальной оболочки и двухслойной модели [2].

Юрий Матвеевич Волчков (д.ф.-м.н., профессор), ведущий научный сотрудник, лаб. механики композитов. 
Зависимость между параметрами модели $h_{1}, h$ и толщиной реальной оболочки $2 H$ устанавливается на основании требования, чтобы поведение модели и реальной оболочки совпадало в двух случаях: при безмоментном напряженном состоянии и при цилиндрическом изгибе. Так, для случая степенного закона ползучести с показателем нелинейности $n$, эти зависимости следующие [2]:

$$
h_{1}=H, \quad h=\left(\frac{n}{1+2 n}\right)^{\frac{n}{n+1}} H .
$$

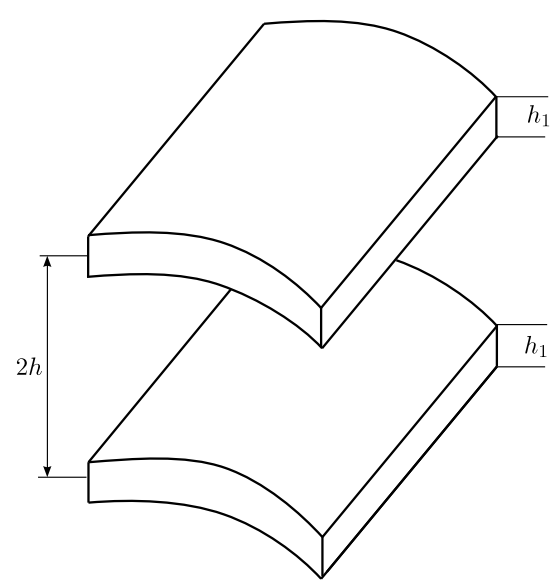

Рис. 1. Двухслойная модель оболочки

При изменении $n$ от 1 до $\infty$ величина

$h$ изменяется в интервале $1 \leqslant h \leqslant H / \sqrt{3}$. Значение $n=\infty$ соответствует случаю идеальной пластичности.

В случае осесимметричного выпучивания круговой цилиндрической оболочки под действием внутреннего давления и сжимающей осевой силы задача сводится к решению следующей системы дифференциальных уравнений, записанной в безразмерных переменных:

$$
\begin{gathered}
m^{\prime \prime}-u\left(\frac{1}{\omega^{+}}+\frac{1}{\omega^{+}}\right)-\frac{2}{\sqrt{3}} \tau+2 p=0 \\
u^{\prime \prime}+(m+\tau) \omega^{+}-(m+\tau) \omega^{-}=0 \\
\left(\omega^{ \pm}\right)^{\frac{2 n}{n-1}}=u^{2}+m^{2}\left(\omega^{ \pm}\right)^{2}
\end{gathered}
$$

где

$$
\omega^{ \pm}=\frac{\sigma_{*} v^{ \pm}}{\varepsilon_{*} s^{ \pm}}, \quad u=\frac{\varepsilon_{2}}{\varepsilon_{*}}, \quad m=\frac{\sqrt{3}}{4} \frac{M_{11}}{h h_{1} \sigma_{*}}, \quad p=-\frac{q_{n} R}{2 h_{1} \sigma_{*}}, \quad \tau=\frac{\sqrt{3}}{4} \frac{T_{11}}{h_{1} \sigma_{*}},
$$

$\varepsilon_{2}$ - скорость окружной деформации ползучести; $M_{11}$ - продольный изгибающий момент; $q_{n}$ - внутреннее давление; $T_{11}$ - продольная сжимающая сила; $v^{+}, s^{+}$и $v^{-}, s^{-}$- интенсивности скоростей ползучести и напряжений в верхнем и нижнем несущих слоях соответственно; $\varepsilon_{*}, \sigma_{*}$ - характерные величины, имеющие размерности скорости ползучести и напряжения соответственно; штрихи обозначают дифференцирование по безразмерной продольной координате $\xi=x / b, b^{2}=4 /(\sqrt{3} R h), R$ - радиус цилиндрической оболочки.

При независимом варьировании величин $u$ и $m$ уравнения (2) являются уравнениями Эйлера для функционала

$$
\begin{aligned}
N=\int_{0}^{l}\left[u^{\prime} m^{\prime}+\frac{1}{2} \psi\left(\omega^{+}\right)+\frac{1}{2} \psi\left(\omega^{-}\right)-(\right. & m+\tau)^{2} \omega^{+}- \\
& \left.-(m-\tau)^{2} \omega^{-}+2\left(\frac{\tau}{\sqrt{3}}-p\right) u\right] d \xi
\end{aligned}
$$


где $\psi(\omega)=\frac{1}{\varepsilon_{*}^{2}} \int \frac{d\left(v^{2}\right)}{\omega}, l=L / b-$ безразмерная длина оболочки.

2. Смешанный вариационный принцип теории ползучести и функционал Работнова. Примем, что в условиях установившейся ползучести напряжения и скорости деформаций ползучести связаны соотношениями

$$
\varepsilon_{i j}=\frac{\partial \Lambda}{\partial \sigma_{i j}}, \quad \sigma_{i j}-\sigma \delta_{i j}=\frac{\partial L}{\partial \varepsilon_{i j}}
$$

где $\Lambda$ - функция напряжений; $L-$ функция скоростей деформаций $\varepsilon_{i j}=(1 / 2) \times$ $\times\left(v_{i, j}+v_{j, i}\right) ; v_{i}$ - компоненты вектора скорости; $i, j \in\{1,2,3\}$.

Пусть тело, занимающее объём $V$, ограниченный поверхностью $S$, находится в состоянии установившейся ползучести под действием поверхностных усилий, заданных на части поверхности $S_{\sigma}$; на части поверхности $S_{v}$ заданы скорости смещений, на части поверхности $S_{\sigma v}$ - некоторые компоненты вектора скорости и тензора напряжений. Запишем функционал

$$
\begin{aligned}
N=\int_{v}\left(\Lambda-\sigma_{i j} \varepsilon_{i j}\right) d V & +\int_{S_{\sigma}} \bar{T}_{i} v_{i} d S+ \\
& +\int_{S_{v}}\left(v_{i}-\bar{v}_{i}\right) T_{i} d S+\int_{S_{\sigma v}}\left[\bar{T}_{\nu} v_{\nu}+\left(v_{\gamma}-\bar{v}_{\gamma}\right) T_{\gamma}\right] d S .
\end{aligned}
$$

Известно, что при независимом варьировании напряжений и скоростей смещений следствием уравнения $\delta N=0$ являются уравнения связи между напряжениями и скоростями смещений, уравнения равновесия и краевые условия.

Можно показать [7], что если принять распределение скоростей деформаций и напряжений в оболочке в соответствии с гипотезами, принятыми при построении двухслойной модели, то функционал (6) преобразуется к функционалу (4).

3. Вычисление критического времени выпучивания цилиндрических оболочек в случае ползучести. Использование функционала (4) в задачах выпучивания оболочек в условиях ползучести позволяет получить выражение для критического времени в явном виде. Момент временем $t^{*}$ называется критическим, если при $t \rightarrow t^{*}$ максимальный прогиб оболочки стремится к бесконечности. Поэтому при определении величин $\omega^{ \pm}$из третьего уравнения системы (2) будем считать, что определяющим является слагаемое, содержащее скорость прогиба:

$$
\omega^{ \pm}=u^{(n-1) / n}\left(1+\varepsilon^{ \pm}\right)
$$

Здесь

$$
\varepsilon^{ \pm}=\frac{n-1}{2 n}(m \pm \tau)^{2} u^{-2 / n}
$$

в случае степенного закона ползучести.

Используем представление (7) в функционале (4) для нахождения коэффициентов $\dot{\alpha}(t)$ и $\beta(t)$, определяющих зависимость от времени пробных функций для скорости смещения $u$ и момента $m$. При вычислении функционала 
будем удерживать величины $\varepsilon^{ \pm}$только в первой степени. Дифференциальные уравнения для определения $\dot{\alpha}(t)$ и $\beta(t)$, которые следуют из уравнений $\partial \bar{N} / \partial \dot{\alpha}(t)=0, \partial \bar{N} / \beta(t)=0$, можно представить в следующем виде:

$$
\begin{gathered}
\alpha(t)=f(\dot{\alpha}(t), \beta(t)), \quad \varphi(\dot{\alpha}(t), \beta(t))=0 \\
(f(\dot{\alpha}(t), \beta(t)) \rightarrow \infty \quad \text { при } \quad \dot{\alpha}(t) \rightarrow \infty) .
\end{gathered}
$$

Вид функций $f$ и $\varphi$ зависит от используемой модели оболочки [4-6].

Общее решение уравнений (8) записывается в виде

$$
t=\int_{q_{0}}^{q} \frac{d f(q, q(\beta))}{q} .
$$

Зависимость $q(\beta)$ определяется из уравнения $\varphi(q, \beta)=0$, константа $q_{0}$ - из условия $\alpha(0)=\alpha_{0}$. Критическое время вычисляется по следующей формуле:

$$
t^{*}=\int_{q_{0}}^{\infty} \frac{d f(q, q(\beta))}{q} .
$$

4. Выпучивание подкрепленных цилиндрических оболочек. В [8] представлены результаты экспериментов по выпучиванию гладких и подкрепленных оболочек из сплава АМг6, кривые кратковременной ползучести которого приведены в [9].

Цилиндрические оболочки длины $L=1200$ мм и радиуса $R=400$ мм были соединены шпангоутами со сферическими днищами. Оболочки были подкреплены продольными и кольцевыми ребрами (в [8] такие оболочки названы «вафельными»). Толщина вафельных оболочек изменялась в пределах от 2 до 2,5 мм; шаг между кольцевыми и продольными ребрами составлял 70 мм, толщина ребер $-8,5$ мм, ширина $-5 \div 8$ мм.

Оболочки нагружались внутренним давлением, затем прилагалось осевое усилие. Температура менялась в пределах от 250 до $300^{\circ} \mathrm{C}$.

Развитие прогиба во времени приводило к формированию кольцевой складки. Форма выпучивания оболочек была близка к осесимметричной. Критическое время определялось моментом катастрофического нарастания прогиба.

В [8] приведены расчётные критические времена для трех оболочек, удовлетворительно согласующиеся с экспериментальными. В расчёте [8] использовались соотношения теории течения и учитывались реальные условия нагрева и нагружения. Форма выпучивания оболочки в расчётах принималась осесимметричной. Краевые условия соответствовали жесткой заделке торцов оболочки у шпангоутов.

Сравнение экспериментальных значений критических времен с расчётными для вафельных оболочек проводилось также в [10]. Подкрепленная оболочка заменялась ортотропной. В расчётах использовались соотношения неустановившейся ползучести по теории течения и соотношения деформационного типа. Константы, входящие в соотношения ползучести, определялись обработкой экспериментальных кривых [9]. Критические времена, вычисленные в [10] по теории течения (они приведены в седьмой колонке таблицы), оказались ближе к экспериментальным, чем вычисленные по деформационной теории. По деформационной теории критические времена на 30-50 \% ниже приведённых. 
В [9] приведены зависимости, с помощью которых аппроксимировались экспериментальные кривые кратковременной ползучести при одноосном растяжении, а также константы, входящие в эти зависимости. На рис. 2, 3 сплошные линии - кривые ползучести, восстановленные нами по аппрокисмационным зависимостям. В масштабе графиков они совпадают с кривыми, приведенными в [9]. На рис. 2, 3 цифры рядом с кривыми ползучести - величины напряжений в мегапаскалях, при которых эти кривые получены в эксперименте. При температуре $T=250^{\circ} \mathrm{C}$ на кривых ползучести имеется достаточно выраженный участок установившейся ползучести. При температуре $T=300^{\circ} \mathrm{C}$ и напряжениях, превышающих $39,2 \mathrm{MПа,} \mathrm{наблюдаются} \mathrm{участки}$ упрочнения. Мы аппроксимировали эти кривые степенным законом установившейся ползучести $\dot{p}=B \sigma^{n}$. При $n=5$ и $B=3,837 \cdot 10^{-28} \mathrm{MПа}^{-n} \cdot \mathrm{ceK}^{-1}$ эти аппроксимации представлены на рис. 2,3 штриховыми линиями.

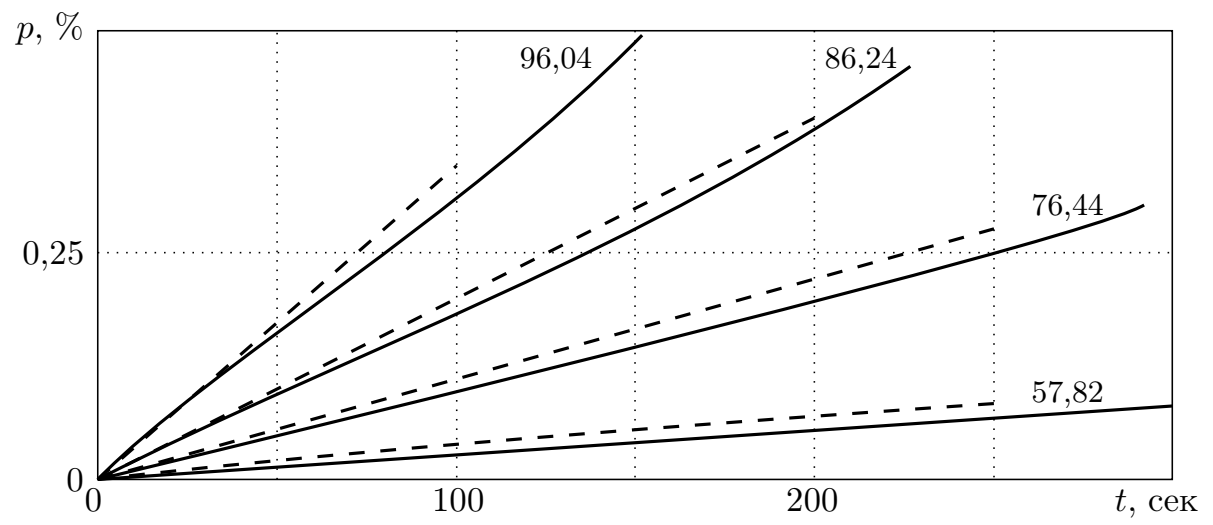

Рис. 2. Кривые кратковременной ползучести сплава АМГ6-М (сплошные линии) и их аппроксимация (штриховые линии) при температуре $T=250^{\circ} \mathrm{C}$

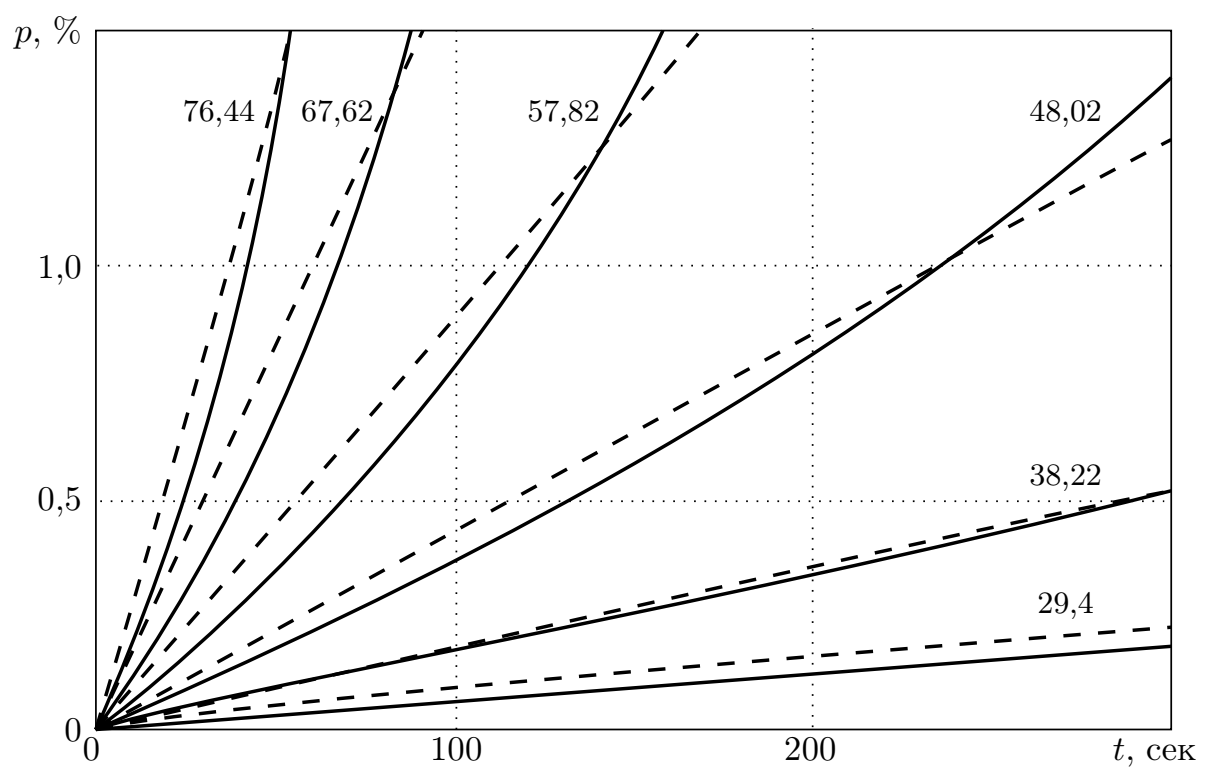

Рис. 3. Кривые кратковременной ползучести сплава АМг6-М (сплошные линии) и их аппроксимация (штриховые линии) при температуре $T=300^{\circ} \mathrm{C}$ 
Вычисления критического времени проводились нами с использованием функционала, построенного для трехслойной модели оболочки [6] и позволяющего вычислять критические времена для подкрепленных оболочек. Приведенные ниже результаты относятся к случаю внутреннего расположения продольных и кольцевых ребер. С учетом характера закрепления торцов оболочки в эксперименте пробные функции задавались в следующем виде:

$$
U=\dot{\theta}\left(t_{2}\right)(1-\cos 2 \pi \xi / l), \quad M=\dot{\beta}\left(t_{2}\right) \cos 2 \pi \xi / l .
$$

Кривые зависимости критического времени от параметра $l$ имеют минимум при некотором значении этого параметра. Соответствующее время считалось критическим временем для длинной оболочки. В данных расчётах величина $l$ соответствовала 5-6 длинам краевого эффекта в упругой оболочке.

В таблице приведено сравнение экспериментальных и расчётных критических времен выпучивания подкрепленных оболочек. В первой колонке приведен номер эксперимента [8], во второй-температура образца, в третьей и четвертой - безразмерные окружное и осевое усилия, в пятой и шестой экспериментальные критические времена, в седьмой - расчётное критическое время по теории течения из работы [10], в восьмой - критическое время $\hat{t}^{*}$, вычисленное с использованием трехслойной модели оболочки и аппроксимации (9), в девятой - погрешность $\hat{t}^{*}$ в процентах по отношению к $t_{\mathrm{exp}}^{*}$. В таблице приняты следующие обозначения [8]: $q^{*}=q R / \delta \sigma_{\mathrm{cr}}, \sigma=N_{x} / 2 \pi R \delta \sigma_{\mathrm{cr}}, q-$ давление, $N_{x}$ - сжимающее усилие, $\sigma_{\mathrm{cr}}=0,605 E \delta / R$ - верхнее критическое напряжение. В [8] приведены два значения экспериментального критического времени: $t_{\text {exp }}^{*}$ - продолжительность действия постоянных по величине осевого усилия, давления и температуры, соответствующих критическому состоянию, $t_{\exp }^{* *}$ - время достижения критического состояния, отсчитанное с момента подачи внутреннего давления.

Сравнение экспериментальных и расчётных критических времен выпучивания вафельных оболочек

\begin{tabular}{c|c|c|c|c|c|c|c|c}
\hline № & $T,{ }^{\circ} \mathrm{C}$ & $q^{*}$ & $\sigma$ & $t_{\text {exp }}^{*}$ сек & $t_{\text {exp }}^{* *}$, сек & $t^{*}$, сек $[10]$ & $t^{*}$, сек & $\%$ \\
\hline 16 & 305 & 0,300 & 0,31 & 24 & 84 & 24 & 28 & +17 \\
18 & 300 & 0,185 & 0,70 & 15 & 68 & 11 & 20 & +35 \\
20 & 250 & 0,400 & 0,38 & 90 & 154 & 121 & 114 & +27 \\
21 & 250 & 0,390 & 0,24 & 346 & 415 & 336 & 418 & +21 \\
22 & 300 & 0,210 & 0,12 & 193 & 228 & 255 & 248 & +29 \\
\hline
\end{tabular}

Заключение. Задачи о деформировании оболочек в условиях ползучести при больших деформациях, к числу которых, в частности, относится задача об определении критического времени, являются сложными геометрически и физически нелинейными задачами. Получение решения таких задач в замкнутом виде на основе полных уравнений не представляется возможным. Следует также учитывать, что константы материала, входящие в определяющие уравнения закона ползучести, в эксперименте определяются с большой погрешностью (порядка 20-30 \%), поэтому применение упрощающих гипотез и приближенных моделей при решении задач ползучести оболочек вполне оправдано. Использование двухслойной модели оболочки Работнова и ее обобщений позволяет эффективно решать задачи о напряженнодеформированном состоянии оболочек в условиях ползучести.

Работа выполнена при поддержке РФФИ (проект № 08-01-00749-а). 


\section{БИБЛИОГРАФИЧЕСКИЙ СПИСОК}

1. Работнов Ю.Н. Приближенная техническая теория упругопластических оболочек // ПMМ, 1951. - № 2. - С. 167-174.

2. Работнов Ю. Н. Ползучесть элементов конструкций. - М.: Наука, 1965. - 752 с.

3. Работнов Ю.Н. О вариационном уравнении установившейся ползучести оболочек // Докл. АН СССР, 1966. - № 2. - С. 300-303.

4. Волчков Ю. М. Осесимметричные задачи ползучести круговых цилиндрических оболочек // Изв. АН СССР. Механика, 1965. - № 5. - С. 118-121.

5. Волчков Ю. М., Немировский Ю. В. Несимметричное выпучивание цилиндрических оболочек в условиях ползучести // Изв. АН СССР. МTТ, 1967. - № 4. - С. 136-138.

6. Волчков Ю. М., Немировский Ю. В. Выпучивание трехслойных цилиндрических оболочек в условиях ползучести // Изв. АН СССР. MTT, 1969. - № 5. - С. 150-158.

7. Волчков Ю. М. Двухслойная модель оболочки Работнова и критическое время выпучивания оболочек при ползучести // ПМТФ, 2010. - Т. 51, № 4. - С. 198-206; англ. пер.: Volchkov Yu. M. Rabotnov's two-layer model of a shell and critical time of shell buckling during creep // J. Appl. Mech. Tech. Phys., 2010. - Vol. 51, No.4. - P. 615-622.

8. Куршин Л. М., Белов В.К., Гусев В. В., Ермаков В. П., Ларионов И.Ф. Влияние кратковременной ползучести на устойчивость гладких и вафельных оболочек // Пробл. прочности, 1975. - № 3. - С. 95-97.

9. Ермаков В. П., Кузнецов А. П. Кратковременная ползучесть сплава АМг6 при одноосном растяжении // ПМТФ, 1972. - № 1. - С. 141-143.

10. Пошивалов В. П. Выпучивание подкрепленных цилиндрических оболочек при ползучести по теории упрочнения // Изв. АН СССР. МТT, 1987. - № 1. - C. 153-158.

Поступила в редакцию 01/III/2010; в окончательном варианте - 08/V/2010.

MSC: 74D10, 74K25

\section{RABOTNOV'S VARIATIONAL EQUATION OF A TWO-LAYER SHELL MODEL AND THE CRITICAL BUCKLING TIME OF REINFORCED SHELLS UNDER CREEP}

\section{Yu. M. Volchkov}

M. A. Lavrentyev Institute of Hydrodynamics, Siberian Branch of RAS, 15, Lavrentyeva pr., Novosibirsk, 630090, Russia.

E-mail: volk@hydro.nsc.ru

Procedure of calculation of critical buckling time of shells under creep with use of Rabotnov's variation equation is presented. Comparison of critical times for the reinforced shells is calculated with use of three-layer model with experimental data and results obtained by other authors is also presented.

Key words: critical buckling time, two-layer shell model, reinforced shells, creep.

Original article submitted 01/III/2010; revision submitted $08 / \mathrm{V} / 2010$.

Yuri M. Volchkov (Dr. Sci. (Phys. \& Math.)), Leading Scientist, Lab. of Mechanics of Composite. 